\title{
Circadian rhythm: A natural, robust, multi-scale control system
}

\author{
Francis J. Doyle III ${ }^{\mathrm{a}, \mathrm{b}, \mathrm{c}, *}$, Rudiyanto Gunawan ${ }^{\mathrm{a}}$, Neda Bagheri ${ }^{\mathrm{c}}$, \\ Henry Mirsky ${ }^{\mathrm{b}}$, Tsz Leung To ${ }^{\mathrm{d}}$ \\ a Department of Chemical Engineering, University of California, Santa Barbara, CA 93106, United States \\ ${ }^{\mathrm{b}}$ Biomolecular Science \& Engineering, University of California, Santa Barbara, CA 93106, United States \\ ${ }^{\mathrm{c}}$ Department of Electrical \& Computer Engineering, University of California, Santa Barbara, CA 93106, United States \\ ${ }^{\mathrm{d}}$ Department of Chemical Engineering, MIT, Cambridge, MA 02139, United States \\ Received 21 February 2006; received in revised form 12 May 2006; accepted 17 May 2006 \\ Available online 17 July 2006
}

\begin{abstract}
The regulatory architecture responsible for robust maintenance of $24 \mathrm{~h}$ cycles is analyzed as a control system. At the gene regulatory level, it is shown that performance attributes, notably phase timing, are controlled in a robust manner. At the next level in the hierarchy, it is shown that synchrony is achieved in populations of neurons to enable clock precision. Finally, at the level of the organism, it is shown that an optimal control approach can be used to reset the clock using a light stimulus.
\end{abstract}

(C) 2006 Elsevier Ltd. All rights reserved.

Keywords: Systems biology; Circadian rhythm; Robustness; Gene regulation

\section{Introduction—circadian clock architecture}

A circadian clock is a highly conserved feature of life on earth, imparting an approximately $24 \mathrm{~h}$ period to many biological behaviors at the cellular, tissular, and organismal levels. They serve to impose internal alignments between different biochemical and physiological oscillations. Their ability to anticipate environmental changes enables organisms to organize their physiology and behavior such that they occur at biologically advantageous times during the day (Edery, 2000) just as visual and mental acuity fluctuate, affecting complex behaviors.

Many of the molecular aspects of the clock have been elucidated for organisms as diverse as Neurospora (Lee, Lores, \& Dunlap, 2000; Merrow, Roenneberg, Macino, \& Franchi, 2001), Arabidopsis (Salom \& McClung, 2004), Drosophila (Williams \& Sehgal, 2001), and mouse (Reppert \& Weaver, 2001, 2002; Shearman et al., 2000). In consequence, models of varying complexity, both deterministic (Forger \& Peskin, 2003; Leloup \& Goldbeter, 1998, 2003; Leloup, Gonze, \& Goldbeter, 1999; Locke, Millar, \& Turner, 2005; Ruoff, Vinsjevik, Monnerjahn, \& Rensing, 2001; Smolen, Baxter, \& Byrne, 2001; Tyson, Hong,

\footnotetext{
* Corresponding author. Tel.: +1 805893 8133; fax: +1 8058934731 .

E-mail address: frank.doyle@icb.ucsb.edu (F.J. Doyle III).
}

Thron, \& Novak, 1999; Ueda, Hagiwara, \& Kitano, 2001) and stochastic (Forger \& Peskin, 2005; Gonze, Halloy, Leloup, \& Goldbeter, 2003), have been proposed. The biological details are coming into sharper focus, as new experiments yield clues to the detailed (and remarkably overlapping) molecular circuitry of both the fly and mammals (Panda, Hogenesch, \& Kay, 2002).

The molecular origins of circadian rhythm are in the functioning of neurons in the suprachiasmic nucleus (SCN), located in the anterior hypothalmus. There are approximately 100,000 neurons in the this region of the brain that operate in synchronized cyclic firing to generate the "master clock" signal that drives other 24-h periods in the body. Various peptides and neuro-transmitters have been studied for their role in circadian synchronization in the $\mathrm{SCN}$, including vasoactive intestinal peptide (VIP), peptide histidine isoleucine (PHI), and gastrin releasing peptide (GRP).

An example of a transcriptional feedback network for circadian rhythm generation can be taken from Drosophila, which is the most heavily studied circadian clock (Hastings, 2000; Reppert \& Weaver, 2000; Young \& Kay, 2001). The core of this clock is shown in Fig. 1 (also, in more detail in Section 3). The transcription rates of the genes period (per) and timeless (tim) are accelerated when protein dClk binds to their promoter regions. The transcribed per and tim messenger RNAs (mRNAs) are exported from the nucleus and translated 

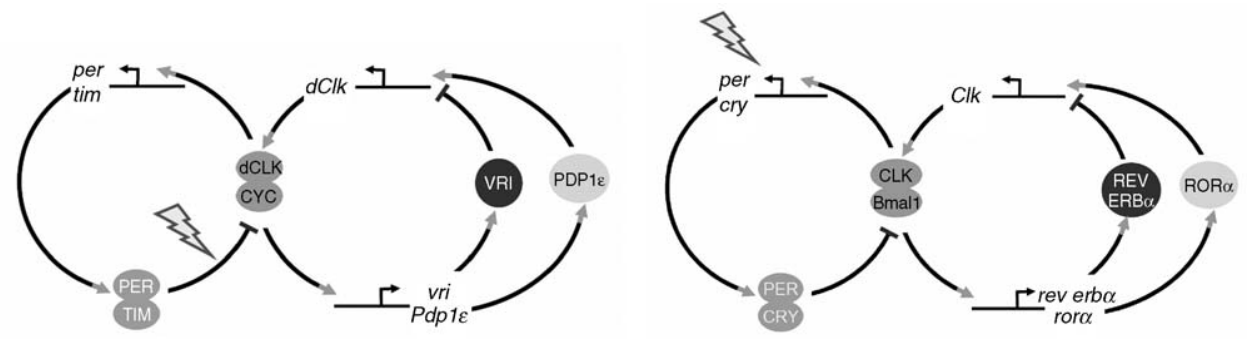

Fig. 1. Circadian gene regulatory architecture for Drosophila (left) and mouse (right).

into proteins PER and TIM, respectively. In the cytoplasm the protein doubletime (DBT) binds to PER. DBT either phosphorylates PER, causing it to be degraded, or allows PER to bind to TIM after a delay, thereby protecting it from degradation. After the DBT-PER-TIM complex is formed, it is imported into the nucleus where it represses the transcription of per and tim and activates the transcription of $d C l k$ (clock). The $d C l k$ mRNA is exported from the nucleus and translated into protein dCLK. Protein dCLK is imported into the nucleus where it represses the transcription of $\mathrm{Clk}$ and activates the transcription of per and tim. This system can be characterized by a two loop transcriptional feedback network, where DBT-PER-TIM negatively feeds back on per and tim transcription and activates $d C l k$ transcription, and dCLK negatively feeds back on $d C l k$ transcription and activates per and tim transcription. In addition to the main (double) negative feedback loop, there are additional loops involving the genes vri and Pdple. This multi-loop architecture is shared by mammals (Fig. 1), although some homologous proteins play different roles (Reppert \& Weaver, 2000). Notable changes include: TIM/PER is replaced by PER/CRY, dCLK by CLK, and the additional circuitry in this case is comprised of VRI and PDPlE. In addition, the mammalian model has light modulation at the transcription step, in contrast with the protein degradation modulation in the fly.

\section{Robust yet fragile architecture}

The coexistence of extreme robustness and fragility constitutes one of the most salient features of highly evolved or designed complexity (Stelling, Sauer, Szallasi, Doyle, \& Doyle, 2004). Optimally robust systems are those that balance their robustness to frequent environmental variations with their coexisting sensitivity to rare events (Morohashi et al., 2002). As a result, robustness and sensitivity analysis are key measures in understanding and controlling system performance.

Robust performance reflects a relative insensitivity to perturbations; it is the persistence of a system's characteristic behavior under perturbations or conditions of uncertainty. Measuring the robustness of a system determines the behavior (the output or performance) as a function of the input (the disturbance) (Ma \& Iglesias, 2002). Formal sensitivity analysis allows the investigation of robustness and fragility properties of mathematical models (Ma \& Iglesias, 2002), yielding local properties with respect to a particular choice of parameter values. Large parametric sensitivity suggests that the system's performance (e.g., periodicity, entrainment, phase-locking) can drastically change with small variations in specific parameters. On the contrary, small sensitivity measures suggest little change in performance. As a result, sensitive elements have evolved as natural control inputs. For instance, experimental data proves that changes in light control phase-resetting properties of the Drosophila melanogaster circadian clock (Myers, Wager-Smith, Rofhenfluh-Hilriker, \& Young, 1996). Meanwhile, elements involved with this light input are some of the most sensitive parameters (Bagheri, Taylor, Stelling, \& Doyle, 2005; Stelling, Gilles, \& Doyle, 2004).

It is expected that the clock is robust, i.e., that perturbations in clock constituents do not radically affect the performance of the clock. Formal sensitivity analysis can be used to reveal robustness in a system and has been used to analyzed these properties in the circadian clock of Drosophila (Stelling and Gilles et al., 2004). Here, a similar analysis is performed on three models of the mouse circadian clock (Forger \& Peskin, 2003; Leloup \& Goldbeter, 2003).

The Leloup and Goldbeter Model "A" (Leloup \& Goldbeter, 2003) uses Michaelis-Menten and Hill-type kinetics to describe the mouse circadian clock in 16 states and 80 parameters. The clock constituents are produced by the three genes per, cry, and Bmall. The protein Bmall activates per and cry and represses Bmall. The proteins PER and CRY dimerize and bind BMAL1, creating an inactive complex that degrades. Phosphorylation of PER, CRY, PER:CRY heterodimer, or BMAL1 enhances degradation, though phosphorylated proteins can be dephosphorylated. So, rising levels of BMAL1 lead to rising levels of PER and CRY. But rising levels of PER and CRY lead to increased sequestration and degradation of BMAL1. Rising levels of BMAL1 also lead directly to lower production of BMAL1.

The Leloup and Goldbeter Model "B" (Leloup \& Goldbeter, 2003) describes the mouse circadian clock in 19 states and 95 parameters and is similar to the above model with several exceptions: (1) there is a fourth gene, rev-erb $\alpha$, whose protein product, REV-ERB $\alpha$, represses expression of Bmall; (2) BMAL1 activates rev-erbo; and (3) BMAL1 has no effect on expression of its gene. Oscillations are produced as in Leloup and Goldbeter Model "A", except that the down-regulation of BMAL1 is accomplished indirectly, by first increasing REV-ERB $\alpha$ levels.

The Forger and Peskin Model (Forger \& Peskin, 2003) uses mass action kinetics to describe the mouse circadian clock in 74 states and 235 parameters. Because many states are equal to zero for all time, the model can be effectively reduced to 62 states and 184 parameters. Though the model does not include BMAL1, as above, it does include two versions of PER (PERI and PER2), 
two versions of CRY (CRY1 and CRY2), as well as REV-ERB $\alpha$. CRY1 and CRY2 repress all system genes, and dimers of REV$\mathrm{ERB} \alpha$ repress the cry genes. Either CRY protein can enter the nucleus only when dimerized to either singly-phosphorylated PER protein; CRY cannot enter the nucleus by itself or when dimerized to un-phosphorylated or doubly-phosphorylated PER. Either PER may be phosphorylated once or twice, but neither may be dephosphorylated. So, rising levels of PERs and CRYs lead to increasing levels of nuclear CRYs and thus lower production of all constituents, including REV-ERB $\alpha$. Since lower REV-ERB $\alpha$ levels lower the repression on the cry genes, this would tend to increase CRY levels. However, the REV-ERB $\alpha$ loop is parameterized at extremely low concentrations, so the loop is effectively absent in the system.

Sensitivity analysis quantifies how much the system behavior (outputs) depends on the parameters that affect the system dynamics. First-order sensitivity coefficients capture the linear dependence of the behavior on these parameters:

$S_{i, j}=\frac{\partial y_{i}}{\partial p_{j}}$

where $S_{i, j}$ is the sensitivity coefficient of the $i$ th system output $y_{i}$ with respect to the $j$ th parameter $p_{j}$. The output $y_{i}$ typically consists of a function of the system states, which in this case include different mRNA and protein concentrations. The circadian rhythm model is represented as coupled ordinary differential equations that produce an asymptotically stable limit cycle:

$\frac{\mathrm{d} \boldsymbol{x}}{\mathrm{d} t}=\boldsymbol{f}(\boldsymbol{x}, t, \boldsymbol{p})$

where $\boldsymbol{x} \in \boldsymbol{R}^{n}$ denotes the state vector, $\boldsymbol{p} \in \boldsymbol{R}^{m}$ the parameter vector, $t$ the time, and $f$ consists of (nonlinear) functions of the states, time, and parameters. There exist numerous methods for computing $S_{i, j}$ from Eq. (2) such as direct differential, finite difference and Green's function (Varma, Morbidelli, \& Wu, 1999).

To assess robustness in each of these models, sensitivity analyses were performed using the BioSens software (Taylor, Gunawan, Gadkar, \& Doyle, in press), which ranks parameter sensitivities according to values generated in the Fisher Information Matrix (FIM). These values were normalized so that the system is most sensitive to parameters with ranks close to zero and least sensitive to parameters with ranks close to one. All three models were analyzed identically, and the conclusions were similar in each case. However, for conciseness, the results are shown for Leloup and Goldbeter Model "B" only.

The robustness inquiry for the Drosophila models (Stelling and Gilles et al., 2004) showed that parameters could be segregated into two broad groups: "Global" parameters, like transcription and translation rates, that affect many processes in addition to the circadian clock, and "local" parameters, like phosphorylation rates, that pertain to the clock exclusively. In addition, a third category of "mixed" parameters, which are neither entirely global nor local, is indicated. It was found that the Drosophila models were sensitive to perturbations in their global parameters but less sensitive to perturbations in their local or mixed parameters. Here, a similar grouping of parameters in the

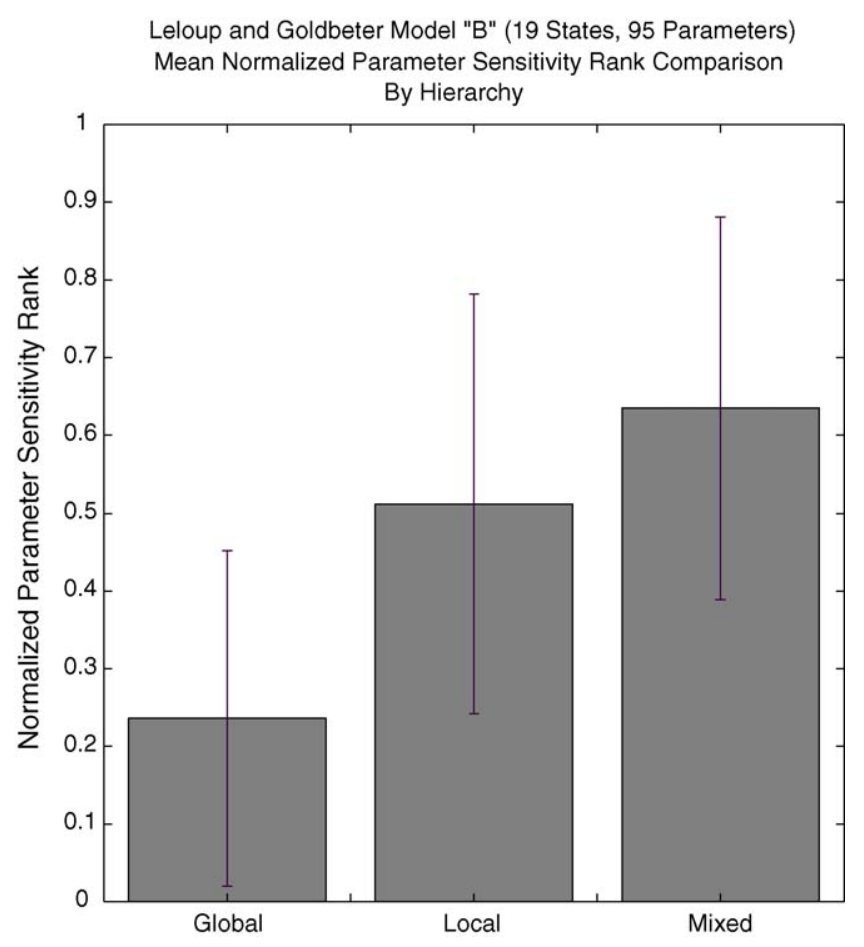

Fig. 2. "Global" and "local" parameter groups. Average normalized ranks for parameter groups in Leloup and Goldbeter Model "B" (19 states, 95 parameters) according to the degree of model sensitivity to parameter perturbations. Normalized ranks near zero indicate model sensitivity to parameter perturbations.

three mouse models yields identical conclusions, i.e., the models exhibit greater sensitivity to perturbation of global parameters than to local or mixed parameters (see Fig. 2).

Again, in an effort to mimic some of the earlier Drosophila analysis (Stelling and Gilles et al., 2004), we allowed each parameter to vary independently, and, provided a stable limit cycle was reached, performed a sensitivity analysis as above; parameter sets not yielding stable limit cycles were discarded. This procedure was repeated 10,000 times for each model, and for each model the mean and standard deviation of the resulting parameter sensitivity ranks was plotted, as shown in Fig. 3. Note that each parameter inhabits a relatively tight sensitivity range, and so the conservation of robustness properties obtained in the earlier paper is also seen here. Interestingly, we were unable to vary the parameter sets as widely for the two Leloup and Goldbeter models as for the Forger and Peskin model without the loss of oscillations, indicating that the Leloup and Goldbeter models are parameterized closer to a bifurcation boundary: in the Leloup and Goldbeter models, parameters were individually dropped to $85 \%$ of their nominal values, left unchanged, or increased to $118 \%$ of their nominal values, but in the Forger and Peskin model parameters were individually dropped to $75 \%$ of their nominal values, left unchanged, or increased to $133 \%$ of their nominal values.

When one plots the sensitivity ranks obtained from perturbation to the nominal parameter sets against the means of the sensitivity ranks obtained from perturbations on the 10,000 Monte Carlo runs, one sees excellent correlations (Fig. 4). Indeed, the correlation value shown in the figure $\left(R^{2}=0.92\right)$ is exceeded 


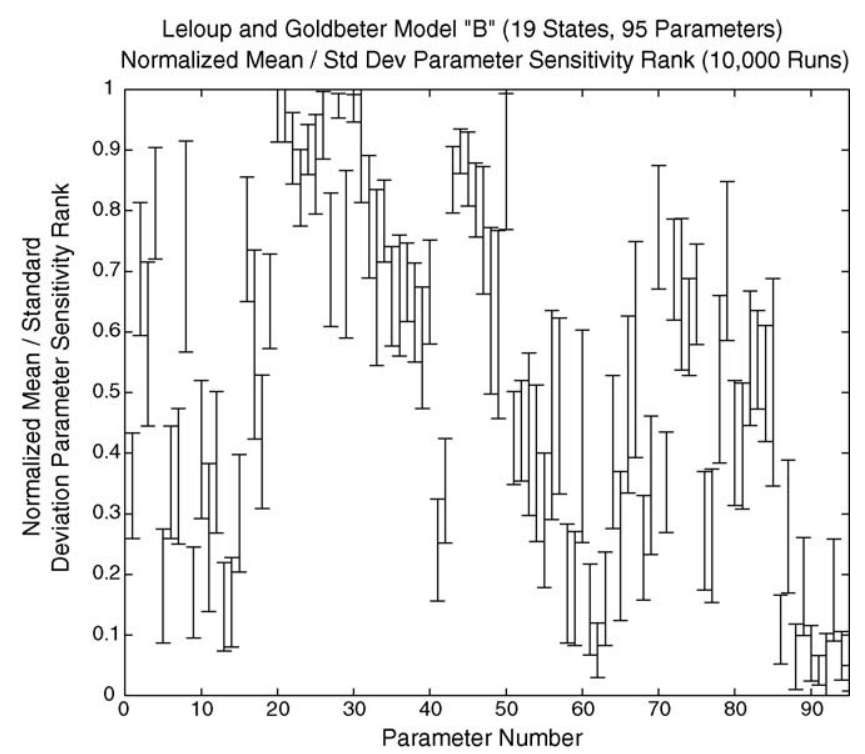

Fig. 3. Conservation of robustness properties. Mean parameter sensitivity ranks and standard deviations as a function of arbitrary parameter number (captured by center of bars, and range, respectively). Sensitivities were determined throughout a section of parameter space yielding oscillations in the model. Parameters were allowed to vary independently. Each parameter took a value at $75 \%$ of nominal, $100 \%$ nominal, or $133 \%$ of nominal. The system was allowed to reach a stable limit cycle and then sensitivity analyses were made by allowing infinitesimal perturbations in each parameter. The sensitivity of the system to each parameter was quantified, ranked and normalized.

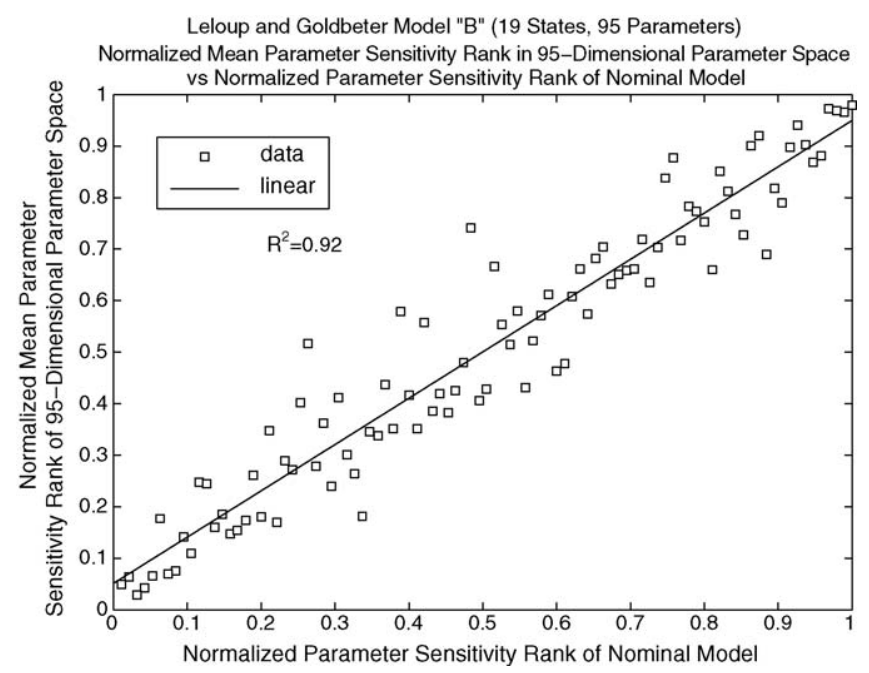

Fig. 4. Similarity of nominal sensitivity rankings and mean sensitivity rankings in 95-dimensional parameter space. Note the high correlation coefficient $\left(R^{2}=0.92\right)$.

by the correlation value for Leloup and Goldbeter Model "A" $\left(R^{2}=0.93\right)$ and the correlation value for the Forger and Peskin Model $\left(R^{2}=0.99\right)$.

\section{Phase behavior and entrainment}

In nature, circadian rhythm does not function independently. This rhythm constantly receives inputs from and synchronizes to the environment. The most obvious and important of environ- mental cues to circadian rhythm is (sun) light, while other common cyclic synchronizing factors include temperature, humidity, meals and social interactions (Johnson, Elliot, Foster, Honma, $\&$ Kronauer, 2004). The ability of circadian rhythm to reset its phase to the external clock (referred to as zeitgebers-literally time givers in German) allows organisms to adapt their activity cycle to best suit the surroundings.

The entrainment (synchronization) property is the most important feature of a circadian rhythm, but the least understood in chronobiology (Johnson, Elliott, \& Foster, 2003; Roenneberg, Daan, \& Merrow, 2003). Here, entrainment refers to an active (dynamic) synchronization response of a free-running oscillator to a cyclic input. To elucidate the mechanisms of circadian entrainment necessitates the understanding of circadian phase behavior. This section presents sensitivity analysis methods for investigating the entrainment mechanisms of a circadian gene network. The methods are applied to the entrainment of Drosophila circadian rhythm to light using a limit cycle model (Leloup \& Goldbeter, 1998).

\subsection{Phase sensitivity}

Phase behavior analysis requires a different approach from the classical sensitivity computations due to the difficulty in representing phase attributes as a function of states. Here, phase represents the time distance (modulo the period) from the reference point to a position on the limit cycle. One enabling concept for quantifying phase is known as the isochron. An isochron of a limit cycle is a set of points (in $\boldsymbol{R}^{n-1}$ ) from which state trajectories evolve to the same phase on the limit cycle. Given two points in the basin of attraction of a limit cycle (not necessarily on the orbit), one can directly compute the phase difference based on the time distance of the isochrons to which these points belong. This phase definition is equivalent to measuring the time difference between two trajectories to reach the same isochron. Thus, the isochrons act as phase grids of a limit cycle as shown in Fig. 5.

The first type of phase sensitivity analysis directly uses the definition of isochrons as an isophasic set of initial conditions. Here, the phase sensitivity to initial conditions can be computed using (Kramer, Rabitz, \& Calo, 1984)

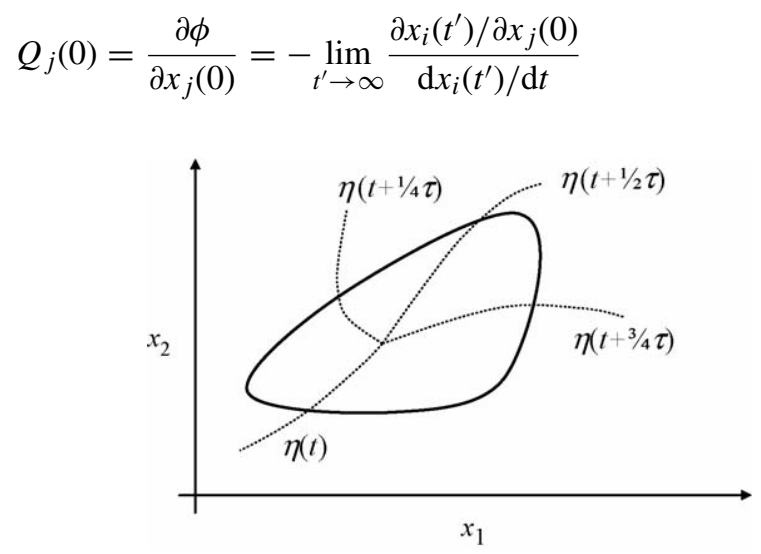

Fig. 5. A hypothetical limit cycle with the isochrons spaced equally in time. The isochrons of an integer multiple of the period apart, are equivalent. 
The numerator can be computed efficiently using the Green's function method. The more interesting phase sensitivity with respect to model parameters can be defined based on the aforementioned sensitivity coefficient. In this case, the parametric phase sensitivity at a given time represents the phase difference between two trajectories starting from the same initial condition; one with the nominal parameter set and the other with the perturbed parameter set (each of the parameters is perturbed independently). Mathematically, this accumulated phase shift can be computed as (Kramer et al., 1984)

$$
\left(\frac{\partial \phi(t)}{\partial p_{j}}\right)_{\eta}=\sum_{i=1}^{n} Q_{i}(t) \frac{\partial x_{i}(t)}{\partial p_{j}}
$$

Note that the period sensitivity is simply the accumulated phase shift after one circle around the limit cycle

$$
\frac{\partial \tau}{\partial p_{j}}=\left(\frac{\partial \phi(t+\tau)}{\partial p_{j}}\right)_{\eta}-\left(\frac{\partial \phi(t)}{\partial p_{j}}\right)_{\eta}
$$

where $t$ is sufficiently large to exclude transient behavior.

In circadian rhythm, the efficacy of an entraining agent depends on the phase at which it is administered. This efficacy is summarized in a phase response curve (PRC) (Johnson, 1999), giving the induced phase shifts as a function of the circadian phase. Modeling of environmental cues can be posed within the framework of the parametric phase sensitivity above (Leloup et al., 1999). In this case, the PRC represents the accumulated phase shift over the duration of external cue (Gunawan \& Doyle, 2005)

$\operatorname{PRC}(t)=\sum_{j=1}^{r}\left[\left(\frac{\partial \phi(t+\Delta \theta)}{\partial p_{j}}\right)_{\eta}-\left(\frac{\partial \phi(t)}{\partial p_{j}}\right)_{\eta}\right] \Delta p_{j}$

where $\Delta \theta$ denotes the (effective) duration of the resetting cue, $r$ is the total number of parameters affected by the entrainment, and $\Delta p_{j}$ represents the magnitude of parameter change due to entrainment.

In an entrained system, the concept of isochorn is no longer applicable, since all points within the basin of attraction will eventually approach the same phase (the system is synchronized). Here, the phase sensitivity can use relative references such as peaks and troughs of the protein and/or mRNA concentrations. In such a case, the phase sensitivity of the peak/trough with respect to different parameters can be derived:

$\frac{\partial t_{e, i}}{\partial p_{j}}=-\left.\frac{\left(\partial f_{i} / \partial \boldsymbol{x}\right)_{p}^{\mathrm{T}}\left(\partial \boldsymbol{x} / \partial p_{j}\right)+\left(\partial f_{i} / \partial p_{j}\right)_{x}}{\left(\partial f_{i} / \partial x\right)_{p}(\partial \boldsymbol{x} / \partial t)}\right|_{t=t_{e, i}}$

where $t_{e, i}$ is the time of the extrema (peak or trough) of the $i$ th state. (The derivation starts from the definition of an extrema $f_{i}\left(\boldsymbol{x}\left(t_{e, i}(\boldsymbol{p}), \boldsymbol{p}\right), p\right)=0$ and continues with the total derivative $\mathrm{d} f_{i}=0$.)

Under entrainment, circadian rhythms maintain a stable phase angle with the external cue, which is a function of the freerunning period (FRP), the period of entrainment and the system phase response (Johnson et al., 2003; Roenneberg et al., 2003). The ability to attain such phase angle hinges on the circadian period that only approximates $24-\mathrm{h}$ (a period of exactly $24 \mathrm{~h}$ will not give a stable phase angle). Furthermore, a stable phase of entrainment is only possible when the FRP does not significantly differ from the period of entrainment, which defines the range of entrainment. In experiments, the phase of entrainment was often obtained as a function of the entraining period, which is known as T-cycle experiment. Although the non-24-h periods have no real natural counterpart, T-cycles have proven useful in elucidating the circadian range of entrainment (Johnson et al., 2004). Using peaks/troughs as phase angle measure, the effect of each parameter variations on the phase of entrainment can be quantified using Eq. (7).

There exist two competing views of circadian entrainment mechanism: continuous (parametric) and discrete (non-parametric) models (Daan, 2000). In parametric entrainment model, light has a continuum effect on the rhythm which is a function of the light intensity. On the other hand, non-parametric model assumes that circadian entrainment is achieved by discrete light signals at dawn and dusk. Each of these models has strengths and weaknesses. Nevertheless, these different views can be consolidated by explaining the circadian entrainment using limit cycle models (Johnson et al., 2003, 2004).

\subsection{Drosophila circadian rhythm phase sensitivity}

The molecular biology of Drosophila circadian rhythm has been extensively studied as a paradigmatic system (Williams \& Sehgal, 2001), which allows for the construction of a mechanistic limit cycle model (Leloup \& Goldbeter, 1998; Leloup et al., 1999). The model captures two negative feedback loops as shown in Fig. 6, which involves 10 states (period and timeless mRNAs, proteins, and their respective phosphorylated

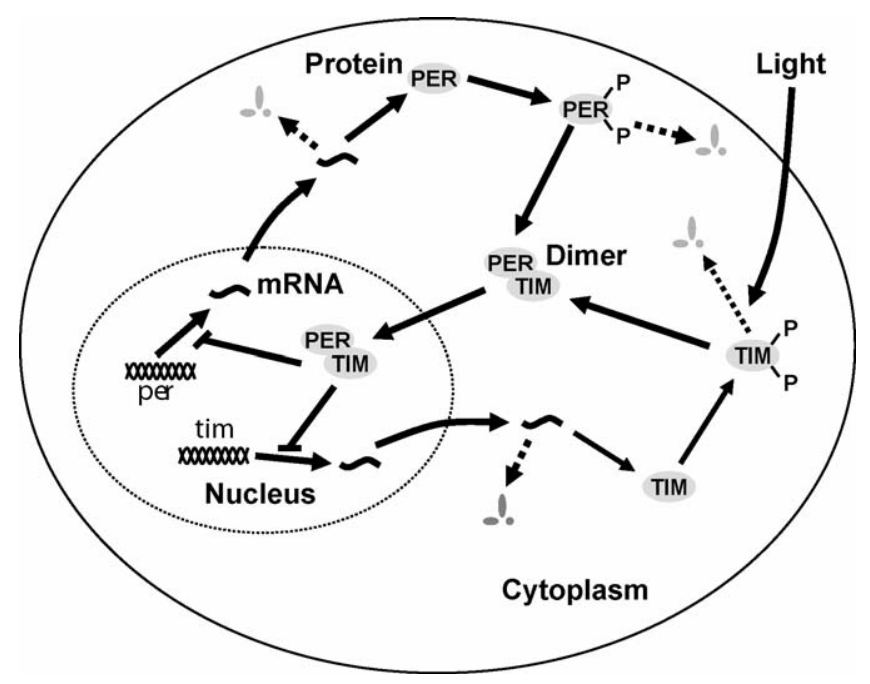

Fig. 6. An overview of Drosophila circadian rhythm gene regulation model (Leloup \& Goldbeter, 1998). The key genes are per and tim which correspondingly produce the proteins PER and TIM. In the cell, the proteins can become prosphorylated and then degraded, or form the dimer PER-TIM which in turn inhibits the transcription of per and tim in the nucleus. Light preferentially enhances the rate of degradation of TIM protein. 
forms, and cytoplasmic and nucleic PER-TIM dimers) and 38 parameters (not shown here for brevity) (Leloup \& Goldbeter, 1998). This model also captures the light input to the circadian rhythm (Zeng, Qian, Myers, \& Rosbash, 1996) and thus provides an example system for studying a circadian entrainment behavior.

Fig. 7a presents the free-running parametric phase sensitivity, which is growing without bound for the parameter variations that affect the FRP. The period sensitivity can be directly computed from Fig. 7a according to Eq. (5). Comparison of the period sensitivity and the phase sensitivity to an impulse parameter perturbation portrays the difference in the set of important parameters from each analysis, as shown in Fig. 7b. This result indicates that the circadian gene architecture separates the control mechanisms that are responsible for active (instantaneous) phase response and for (long-term) period modulation.
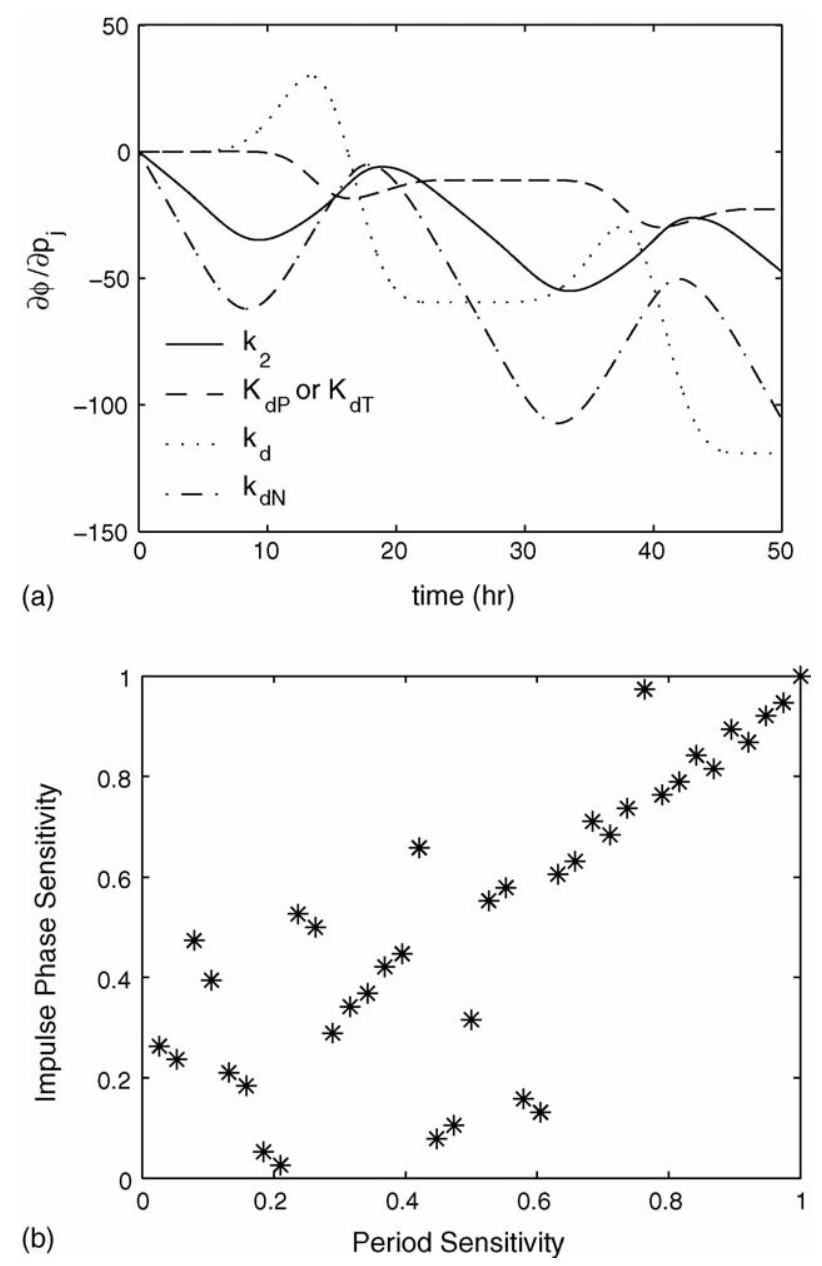

Fig. 7. (a) Parametric phase sensitivities of the 10-state mechanistic Drosophila circadian rhythm model (for brevity, only 5 parameters are shown here). The parameters $k_{2}, K_{\mathrm{d} P}\left(K_{\mathrm{d}} T\right), k_{\mathrm{d}}$, and $k_{\mathrm{d} N}$ refer to the nuclear transport constant of the dimer PER-TIM, the Michaelis-Menten constant of PER (TIM) degradation, the degradation rate constant of proteins, and the degradation rate constant of nuclear dimer, respectively, (b) Comparison of the period sensitivity and the phase sensitivity to impulse parameter perturbations (i.e., the slopes of the curves in (a)) based on the ranking of the maximum sensitivity magnitudes. The higher indexes correspond lower sensitivities.
The control mechanisms of circadian entrainment to light are studied as a function of the length of the day and the period of entrainment (T-cycle). Using continuous photoperiod (parametric model), Fig. 8 indicates the strong correlations between the period and the phase angle sensitivities to parameters. (The model failed to entrain at day length of $20 \mathrm{~h}$.) Such correlations suggest that the entrainment to continuous photoperiod entails the modulation of the underlying circadian period. In experiments, post-entrainment FRP of Drosophila activity differed from that before the entrainment (usually referred to as after-effects) (Kumar \& Sharma, 2004), confirming the aforementioned observation.

In T-cycle study, the quantity of interest is the slope of the phase angle with respect to the period, i.e., the parametric sensitivity:

$$
\frac{\partial}{\partial p_{j}}\left(\frac{\mathrm{d} t_{e, i}}{\mathrm{~d} T}\right)
$$

where $T$ is the entraining period. Again, the entrained system shows strong correlation between the period sensitivity and the T-cycle phase sensitivity (results not shown), which gives support for the importance of period modulation in continuous photoperiod entrainment.

In the literature, a skeleton photoperiod consisting of two light pulses is also known to entrain circadian rhythms (Johnson et al., 2004). These experiments gave support for the nonparametric model of entrainment. Fig. 9 presents a comparison between the period and the skeleton phase angle sensitivity. The correlations between the two analysis were inferior to the continuous photoperiod analysis, in which the average deviation from the period sensitivity ranking is doubled to that of Fig. 8. The difference between Figs. 8 and 9 arises from the nature of light entrainment in the two photoperiods. In the skeleton photoperiod, light pulses only induce a transient phase response from the system, and the system quickly returns to its endogeneous limit cycle. On the other hand, the constant light input during day in the continuous photoperiod drives the system, albeit temporarily, to another limit cycle corresponding to a circadian rhythm under 24-h daylight condition. Such response correlates better with a long-term period modulation by light.

\section{Multicellular scale-synchronization}

We have begun to investigate the properties of robust time keeping at the level of populations of neurons in the SCN. It has been shown recently (Herzog, Aton, Numano, Sakaki, \& Tei, 2004) that the "precision" in timekeeping emerges only at the level of interconnected populations of neurons. Such "synchrony" has been studied in many other contexts, and the mathematical details are beginning to emerge in the circadian neuron networks.

In Drosophila, robust self-sustained oscillations persist for weeks in constant darkness. The neuropeptide pigmentdispersing factor (PDF) is required to maintain behavioral rhythms under constant darkness. In addition, PDF is required 


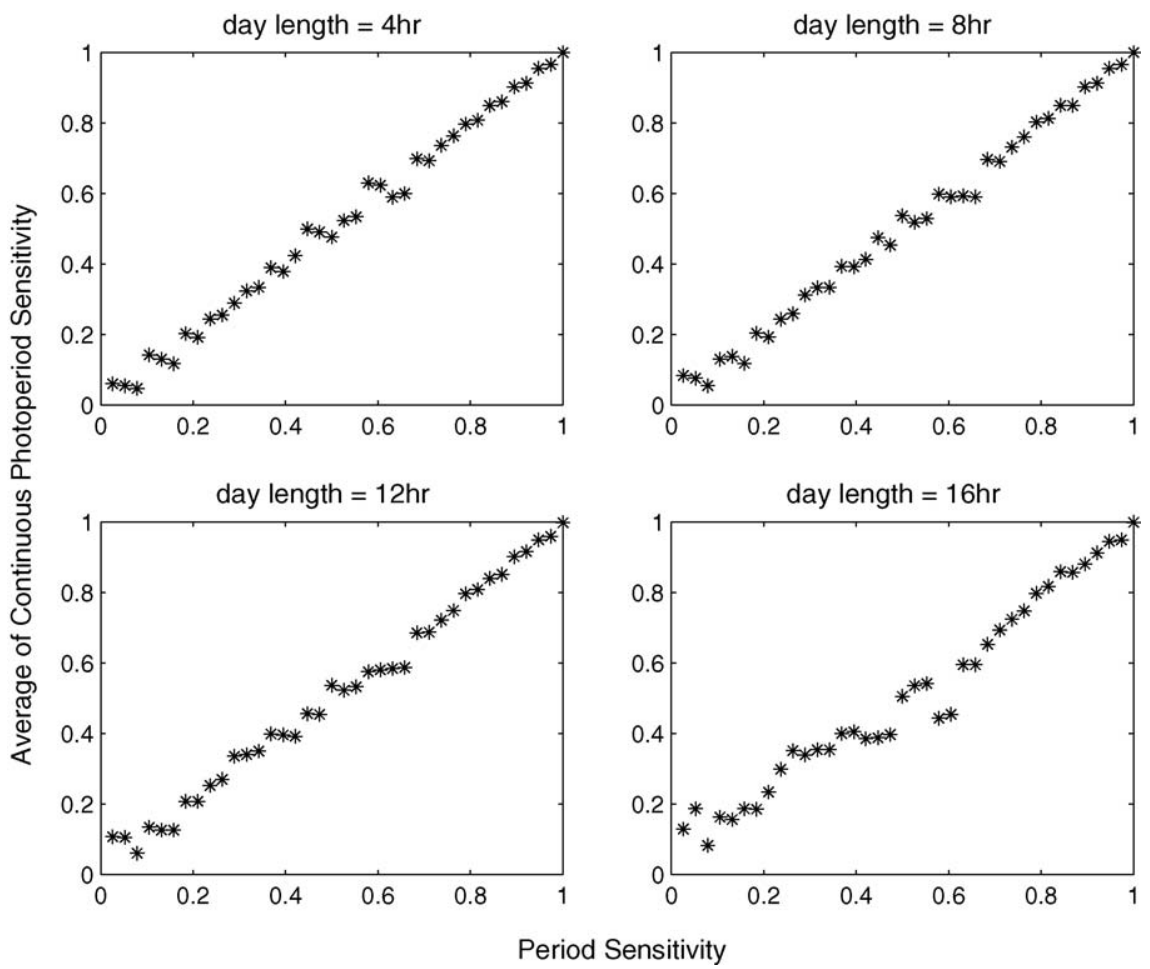

Fig. 8. Comparison between the period and phase of entrainment sensitivity in continuous photoperiod (LD, light-dark) entrainment. The phase angle sensitivity ranking is averaged over the peak and trough sensitivity of all the states in the system.

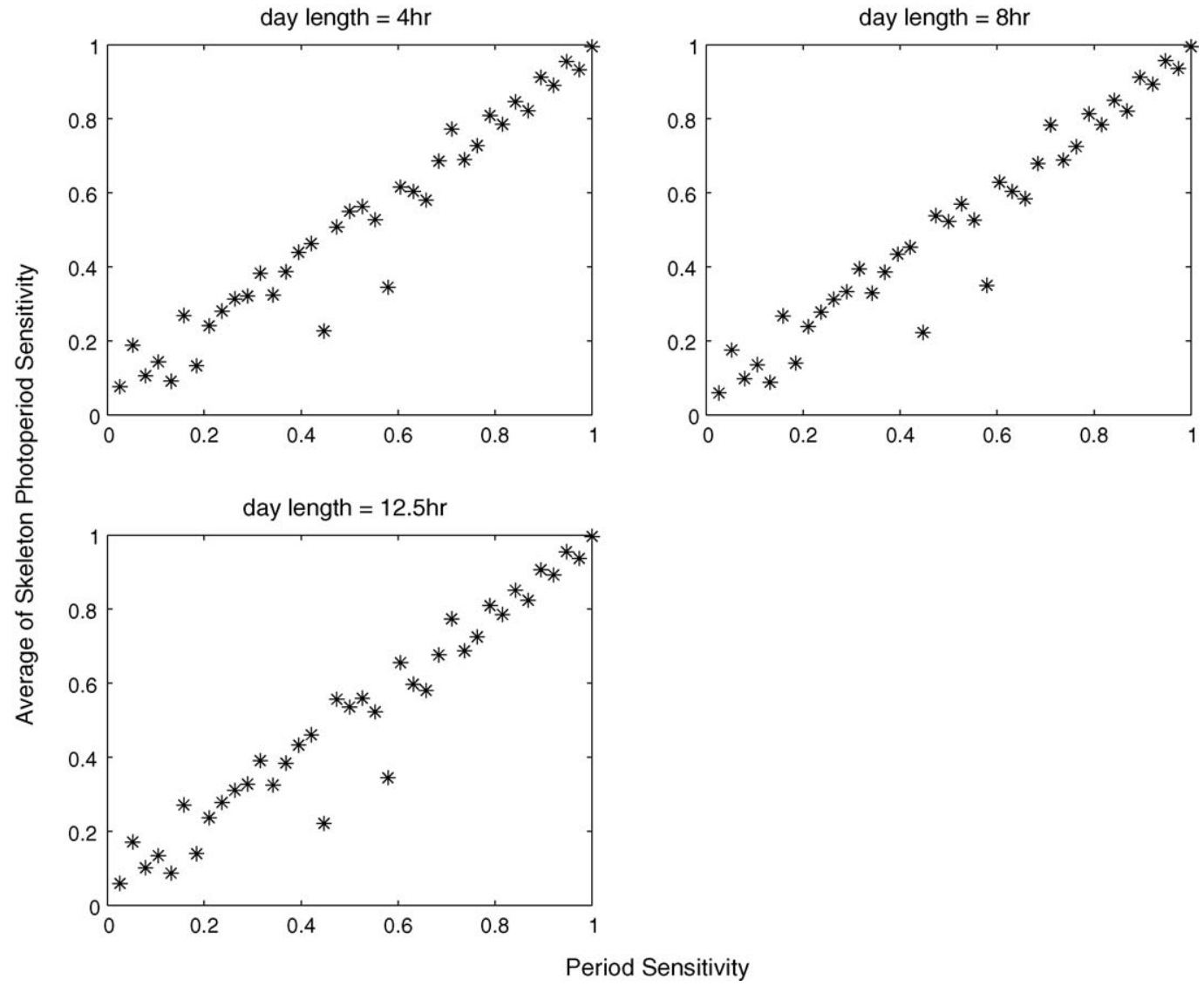

Fig. 9. Comparison between the period and phase of entrainment sensitivity in skeleton photoperiod entrainment. The phase angle sensitivity ranking is averaged over the peak and trough sensitivity of all the states in the system. In skeleton photoperiod, the system treats the shorter of the two dark phases as subjective day, and thus the analyses are unique up to $12 \mathrm{~h}$ day length. 
to synchronize the phase and amplitude of rhythms among the diverse pacemakers (Helfrich-Förster, 2005). PDF is found in the s-LNv and 1-LNv and its released is rhythmic (Park et al., 2000). It has been speculated that PDF acts on the input side of the clock cells (Petri \& Stengl, 1997). Normally, neu-ropeptides G-protein coupled receptors (GPCRs) (Hewes \& Taghert, 2001) and GPCRs activate cAMP or $\mathrm{Ca} 2+$ singaling pathways. PKC mutants exhibit arrhythmic activity (Park et al., 2000).

Our preliminary studies are based on the 10-state Drosophila gene regulation model by (Leloup \& Goldbeter, 1998). The full mathematical details are available in (To, Henson, \& Doyle, 2005). A key assumption in this model is that the signaling agent responsible for coupling in Drosophila (PDF), acts independently of light. Light was assumed to have no effect on PDF. In other words, light was only responsible for TIM degradation, while PDF was responsible for inducing per transcription. The release of PDF was in phase with per transcript.

The synchronization characteristics are evidenced in Fig. 10, where 100 neurons are simulated. In order to generate a range of basal clock times (uncoupled), the basal transcription rate of per $v_{S P 0}$ was subjected to zero mean normally distributed perturbations with standard deviation of $25 \%$. With such manipulation, only approximately $40 \%$ of all neurons displayed circadian rhythms without PDF signaling (top plot, Fig. 10). In order to incorporate variations in free-running periods, zero mean normally distributed perturbation with standard deviation of $10 \%$ was applied to the $k_{s} P$, the translation rates of PER. As in the original gene regulation model, PER and TIM were assumed to be fully symmetrical. Hence, the rates of transcription of per and tim, as well as the rates of translation of PER and TIM,
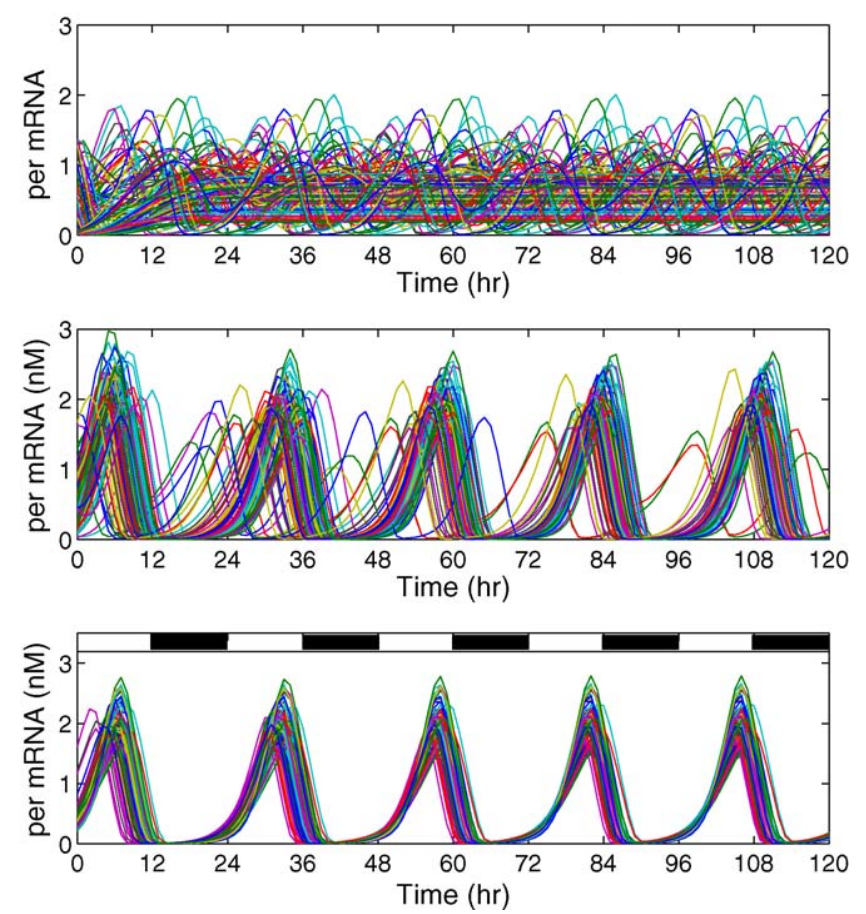

Fig. 10. Synchronization of 100 Drosophila circadian oscillators: (top) uncoupled, constant darkness conditions, (ii) coupled constant darkness conditions, (iii) coupled, alternating light-dark conditions.
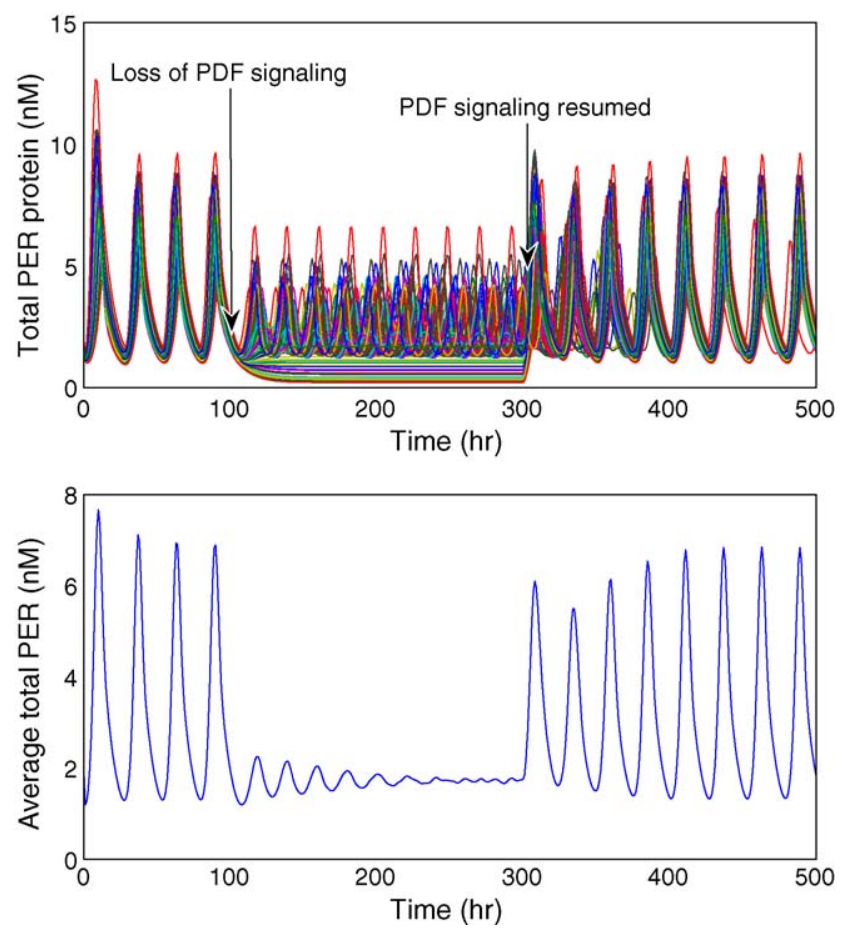

Fig. 11. Effect of eliminating PDF signaling on the synchronization dynamics of the 100-cell ensemble.

were set to be equal $\left(v_{S T}=v_{S P}\right.$ and $\left.k_{S T}=k_{S P}\right)$. The middle plot shows the entraining influence of the coupling peptide. The bottom plot shows the entrainment of 100 circadian oscillators by a 12:12 h light dark cycle. The light dark cycle was generated by manipulating the rate of TIM degradation as a square wave: $v_{\mathrm{d} T}=2$ during the dark phase and $v_{\mathrm{d} T}=4$ during the light phase.

The impact of the coupling agent is particularly striking in the results depicted in Fig. 11. A simulated blocking of the receptor corresponding to the coupling protein is carried out. At a time of $100 \mathrm{~h}$, the "block" was introduced and the ensuing asynchrony is apparent. Once the signaling is resumed $(t=300 \mathrm{~h})$, the synchrony is restored.

Work is underway to quantify the robustness of the population behavior under perturbations in the transcription process, as well as environmental stimuli.

\section{Control insights}

\subsection{Resetting the clock}

Using control engineering parlance, one may refer to the elements that influence or entrain the circadian oscillator (zeitgebers) as manipulations, and the elements that exhibit quantifiable circadian rhythms as measurements. The use of this terminology is reflective of the spirit of the work described in this section in looking for ways to influence rhythms to fine tune physiological performance. Continuing the control analogy, the open-loop characteristics (i.e., with no intervention) of the typical human are to adjust at a rate of approximately $60-90 \mathrm{~min}$ of phase per day (Lamberg, 1994). In other words, jet lag accommodation 

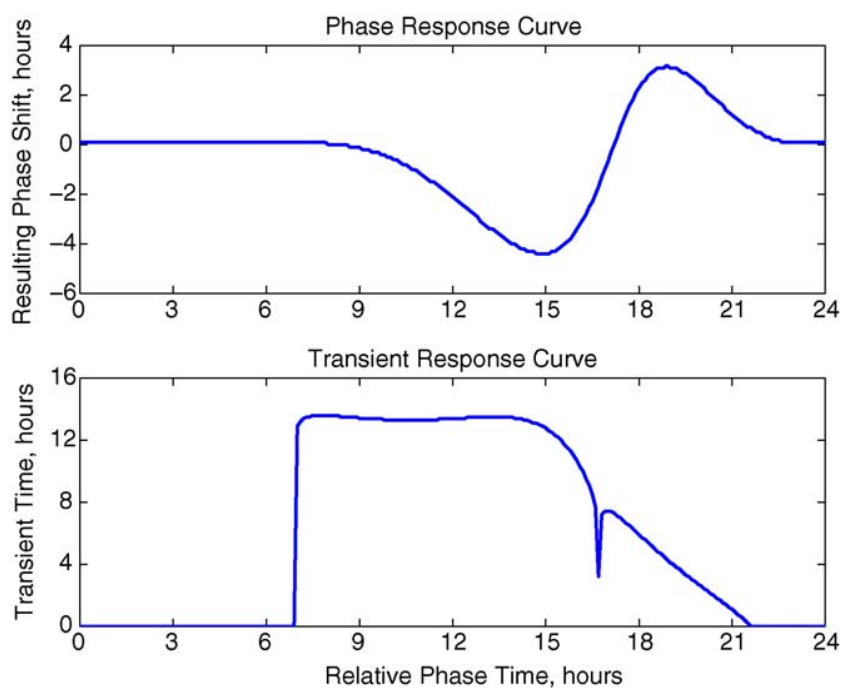

Fig. 12. Phase and transient response curves of the Drosophila circadian model. (Top) A one-minute light pulse injected at various times throughout an oscillation results in a corresponding phase shift, captured by the PRC. The maximum phase advance from a single stimulus is $3.2-\mathrm{h}$, while the maximum phase delay is $4.6-\mathrm{h}$. (Bottom) Transient response dynamics of the model resulting from a 1-minute light pulse applied at various times within a period of oscillation are depicted on the vertical axis. The horizontal relative time axis corresponds directly to that of the PRC.

occurs at a rate of approximately 2-3 days for a 3-h time zone change. Westward travel is slightly easier, as the natural freerunning human circadian clock (i.e., in absence of light) has a period of approximately $25 \mathrm{~h}$.

From a control perspective, there are two important attributes of the resetting response characteristics: the phase shift (captured by the phase response curve (PRC), and the associated transient, which we define via the transient response curve (TRC). These ideas are illustrated in Fig. 12.

\subsection{Control design}

Although period and timeless genes and proteins undergo similar oscillations, TIM is known to be unstable in the presence of light. Light-induced TIM degradation requires neither the involvement of PER, nor any other functional clock protein, and is considered a direct control input pathway that catalyzes entrainment. The Leloup and Goldbeter mathematical model assumes that a 1-min light pulse doubles the maximum TIM degradation rate over a 3-h time interval (Leloup \& Goldbeter, 2000).

Phase-resetting control is accomplished through use of an optimal control algorithm (Khalil, 2002; Stunners, 1998) with a cost function, $C(k)$, spanning a single period of oscillation, $\tau$. The cost function accounts for both the accuracy of the system phase and the "cost" of the control input (light pulse). As a result, the controller optimizes the light timing by minimizing $\tau_{\text {delay }}, \tau_{\text {trans }}$, and $\left(\phi-\phi^{+}\right)$, suitably weighted: $\omega_{\text {delay }}, \omega_{\text {trans }}$, and $\omega_{\phi} \cdot \tau_{\text {delay }}$ is the delay associated with a light pulse at time $k, \tau_{\text {trans }}$ is the transient response (from the TRC) associated with a light pulse at time $k$, and $\left(\phi-\phi^{+}\right)$is the phase difference, measured in units of time, between the reference and system models. This weighted objective includes penalties for both tracking (last term) as well as speed of response (second term). The expressions are detailed below:

$$
\begin{aligned}
C(k) & =\eta^{\mathrm{T}}(k) \boldsymbol{Q} \eta(k), k \in[t, t+\tau) \\
\eta(k) & =\left[\begin{array}{lcc}
\tau_{\text {delay }} & \tau_{\text {trans }} & \left(\phi-\phi^{+}\right)
\end{array}\right]^{\mathrm{T}} \\
\boldsymbol{Q} & =\left[\begin{array}{ccc}
\omega_{\text {delay }} & 0 & 0 \\
0 & \omega_{\text {trans }} & 0 \\
0 & 0 & \omega_{\phi}
\end{array}\right]
\end{aligned}
$$

The first entry in $\eta$ defines the time interval between the current time and the relative time at which the control may be applied.

$$
\tau_{\text {delay }}= \begin{cases}k-t & \text { if } k \geq t \\ k-t+\tau, & \text { if } k<t\end{cases}
$$

A complement of the PRC, the transient response curve (TRC) defines the amount of time it takes for the system output trajectory to return to its stable limit cycle upon light stimulus (Fig. 12, bottom plot). Eq. (11) describes the TRC function as mapping a 1-minute light input occurring at relative time $k$; to a measure of the ensuing transient period.

$\tau_{\text {trans }}=g_{\text {trc }}(k)$

Similarly, Eq. (12) describes the PRC function as mapping the same light pulse applied at relative time $k$ to the incurred phase shift, $\phi^{+}$, allowing the system to minimize the phase difference, $\phi$, through admission of a series of light pulses.

$\phi^{+}=g_{\text {prc }}(k)$

Minimizing the cost function by choosing an optimal $k$ (where $k=[t, t+\tau)$ ) yields the optimal control time $\tilde{k}$ along with its respective time delay, $\tilde{\tau}_{\text {delay }}$, and transient period, $\tilde{\tau}_{\text {trans }}$. The real-time control input occurs once time $t$ is equal to $\tilde{k}$.

\subsection{Results}

For simplicity, the system is studied in a constant dark environment with a one-minute light pulse as the controlling agent. Using the weight $\boldsymbol{Q}=\operatorname{diag}(1,1,1000)$, the controller is able to recover approximately 12 -h of phase difference with 0.2 $\mathrm{h}$ accuracy in approximately three and half days, as shown in Figs. 13 and 14.

Performance functions are varied in order to optimize specific criteria. When transient weights are greater, the system recovers phase almost immediately after the light input has terminated. When time delay weights are greater, the control input is applied sooner, but the total recovery time suffers as it takes the system longer to track the reference.

Phase resetting dynamics are captured in the recovery plot (Fig. 14). The phase recovery time is greater for negative initial phase differences. This discrepancy evolves from the fact that the PRC is asymmetric for positive and negative phase shifts. The maximum achievable phase delay by a single light pulse is $4.6-\mathrm{h}$, whereas the maximum phase advance is $3.2-\mathrm{h}$. 

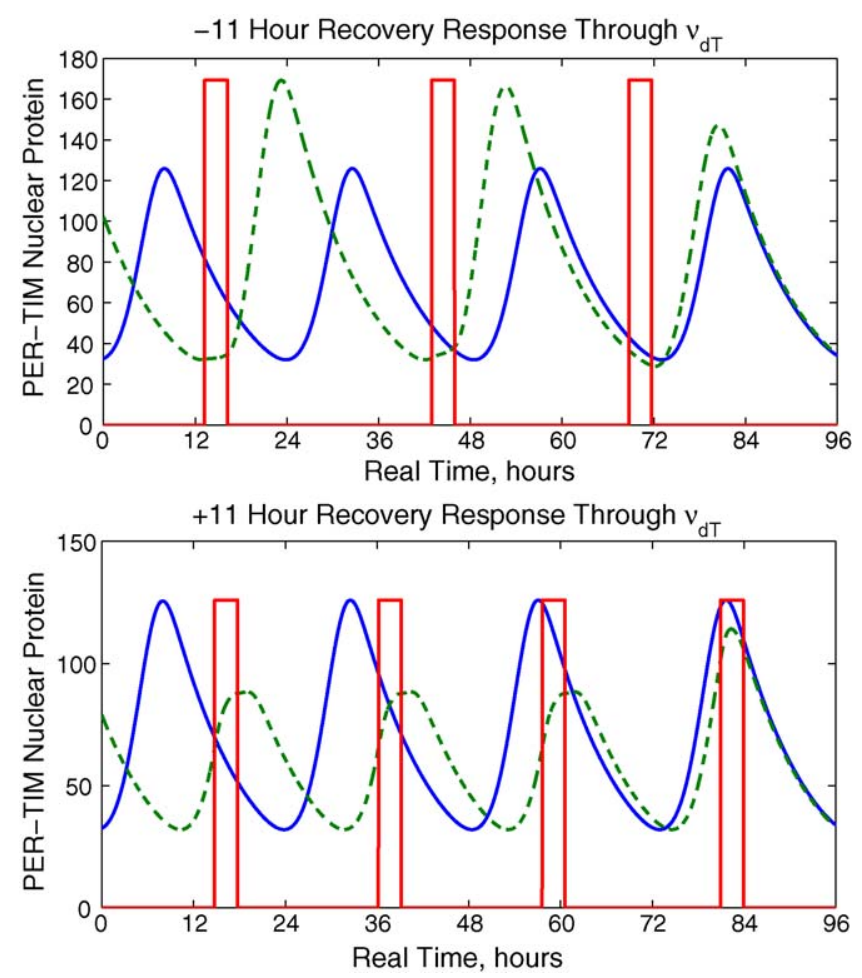

Fig. 13. Optimal phase-tracking of the Drosophila circadian oscillator. Control inputs are depicted as square waves indicating the time and duration of lightinduced TIM degradation. This light impulse corrects for an +11.5 -h (upper simulation) and -11.5 -h (lower simulation) initial phase difference, terminating control once the difference in phase is less than or equal to 0.2 -h. The reference is depicted through a solid line oscillation while the controlled signal is represented by the dashed line.

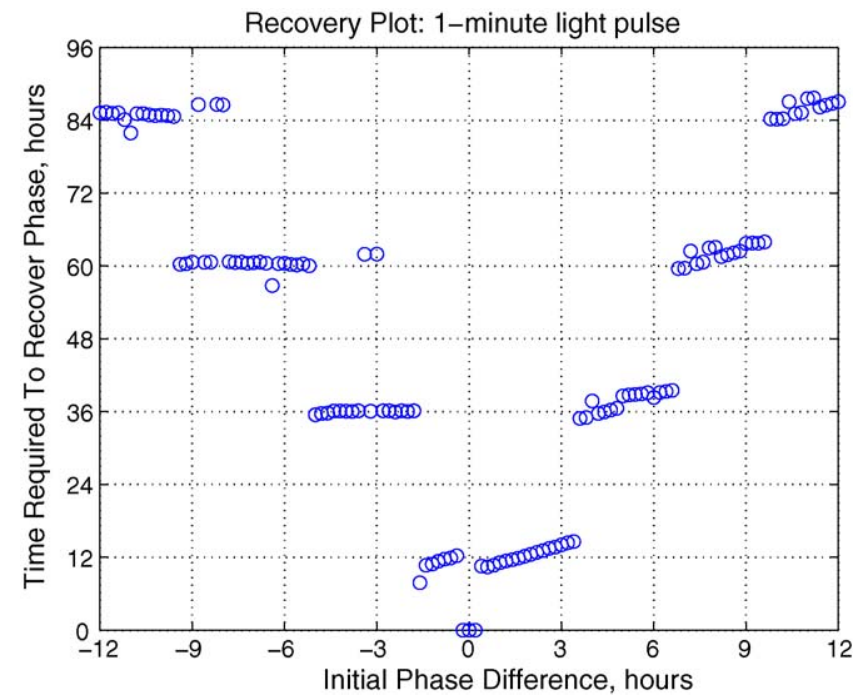

Fig. 14. The Drosophila circadian recovery plot. The asymmetry of the recovery time relates directly to the discrepancy between the maximum phase delay vs. the maximum phase advance with respect to identical control inputs. Initial phase differences ranging from -12 to $+12 \mathrm{~h}$ are reduced to a maximum difference of $0.2-\mathrm{h}$, after which the signals are considered in phase. This data was constructed from detailed simulations of 97-runs with initial phase difference intervals of 0.25-h.
As a result, capturing a 12-h phase difference may be more efficient by forcing phase delays rather than advances. The apparent "outliers" reflect the tradeoffs between the phase response and the transient response terms and are affected by the tuning weights.

\subsection{Discussion}

The combination of robustness and sensitivity allow biological systems to adapt to their environments. For instance, jet lag occurs as a result of robust circadian properties that maintain the synchrony between biological clocks and their natural environment. Concurrently, jet lag diminishes due to the system's sensitivity toward light cues; a change in light patterns resets the circadian phase. Therefore, one may control the biological clock through pulses of light and alleviate circadian related disorders or assist individuals working in rotating shifts. Circadian disorders include chronic sleep disorders in the elderly, manic-depression, and seasonal affective disorders (Edery, 2000). Controlling the circadian clock may also help nurses on a repetitive shift work schedule who are two- to threefold more likely to misdiagnose and wrongly treat patients than their daytime counterparts (Gold et al., 1992). As a result, controlling the circadian clock may reduce the risk of accidents related to the ill effects of "un-natural" work schedules, some of which include the Chernobyl nuclear plant (1986), the chemical explosion of the Union Carbide plant (1984), and the grounding the oil tanker Exxon Valdez (1989) as a few examples (Lamberg, 1994).

\section{Summary}

The generation of circadian rhythms in organisms as simple as Drosophila and as complex as a mammal are remarkable paradigms of robust hierarchical control. The structures, at the gene regulation level, are largely preserved, and the robust performance characteristics (both at the single cell level, and at the ensemble level) are nothing short of amazing.

Through detailed analyses of the control theoretic properties of these circuits, we aim to: (i) shed light on the design principles that underly the circadian clocks; and (ii) through this understanding, point to promising directions for therapeutic interventions for medical problems as well as optimized performance for civilian and military applications.

\section{Acknowledgments}

Sections of this manuscript describe projects that are ongoing collaborations of FJD with the following individuals: Jörg Stelling (ETH), Mike Henson (U. Massachusetts), Erik Herzog (Washington U.), and Linda Petzold (UCSB) — their intellectual contributions are greatly appreciated. This work has been supported by research funds from DARPA, the Air Force Office of Scientific Research, and by the Institute for Collaborative Biotechnologies through grant DAAD19-03-D-0004 from the U.S. Army Research Office. 


\section{References}

Bagheri, N., Taylor, S., Stelling, J., \& Doyle, F. J., III. (2005). Phase sensitivity analysis of biological oscillators. In Proceedings of Foundations of Systems Biology and Engineering.

Daan, S. (2000). The Colin S. Pittendrigh Lecture. Colin Pittendrigh, Jrgen Aschoff, and the natural entrainment of circadian systems. Journal of Biological Rhythms, 15(3), 195-207.

Edery, I. (2000). Circadian rhythms in a nutshell. Physiological Genomics, 3(2), $59-74$.

Forger, D. B., \& Peskin, C. S. (2003). A detailed predictive model of the mammalian circadian clock. Proceeding of the National Academy of Sciences of the USA, 100, 14806-14811.

Forger, D. B., \& Peskin, C. S. (2005). Stochastic simulation of the mammalian circadian clock. Proceeding of the National Academy of Sciences of the USA, 102(2), 321-324 [Online]. Available: http://dx.doi.org/10.1073/pnas.0408465102.

Gold, D., Rogacz, S., Bock, N., Tosteson, T. D., Baum, T. M., Speizer, F. E., et al. (1992). Rotating shift work, sleep, and accidents related to sleepiness in hospital nurses. American Journal of Public Health, 82, 10111014.

Gonze, D., Halloy, J., Leloup, J.-C., \& Goldbeter, A. (2003). Stochastic models for circadian rhythms: Effect of molecular noise on periodic and chaotic behavior. Comptes rendus Biologies, 326, 189-203.

Gunawan, R., \& Doyle, F. J., III. (2005). Phase sensitivity analysis of a circadian gene network. In Proceedings of 44th IEEE Conference on Decision and Control.

Hastings, M. (2000). Circadian clockworks: Two loops are better than one. Nature Reviews Neuroscience, 1, 143-146.

Helfrich-Förster, C. (2005). Neurobiology of the fruit fly's circadian rhythm. Genes, Brain and Behavior, 4, 65-76.

Herzog, E., Aton, S., Numano, R., Sakaki, Y., \& Tei, H. (2004). Temporal precision in the mammalian circadian system: A reliable clock from less reliable neurons. Journal of Biological Rhythms, 19, 35-46.

Hewes, R., \& Taghert, P. (2001). Neuropeptides and neuropeptide receptors in the Drosophila melanogaster genome. Genome Research, 11, 1126-1142.

Johnson, C. H. (1999). Forty years of PRCs-What have we learned? Chronobiology International, 16(6), 711-743.

Johnson, C. H., Elliott, J. A., \& Foster, R. (2003). Entrainment of circadian programs. Chronobiology International, 20(5), 741-774.

Johnson, C. H., Elliot, J., Foster, R., Honma, K., \& Kronauer, R. (2004). Fundamental properties of circadian rhythms. In J. C. Dunlap, J. J. Lores, \& P. J. DeCoursey (Eds.), Chronobiology: Biological timekeeping (pp. 67-105). Sunderland, MA: Sinauer Associates Inc.

Khalil, H. K. (2002). Nonlinear systems. Upper Saddle River, NJ: Prentice Hall.

Kramer, M. A., Rabitz, H., \& Calo, J. M. (1984). Sensitivity analysis of oscillatory systems. Applied Mathematical Modelling, 8, 328-340.

Kumar, S., \& Sharma, V. K. (2004). Entrainment properties of the locomotor activity rhythm of Drosophila melanogaster under different photoperiodic regimens. Biological Rhythm Research, 35, 377-388.

Lamberg, L. (1994). Bodyrhythms: Chronobiology and peak performance. New York, NY: William Morrow \& Co.

Lee, K., Lores, J. J., \& Dunlap, J. C. (2000). Interconnected feedback loops in the Neurospora circadian system. Science, 289(5476), 107110

Leloup, J.-C., \& Goldbeter, A. (1998). A model for circadian rhythms in Drosophila incorporating the formation of a complex between the PER and TIM proteins. Journal of Biological Rhythms, 13, 70-87.

Leloup, J.-C., \& Goldbeter, A. (2000). Modeling the molecular regulatory mechanism of circadian rhythms in Drosophila. BioEssays, 22(1), 84-93.

Leloup, J.-C., \& Goldbeter, A. (2003). Toward a detailed computational model for the mammalian circadian clock. Proceeding of the National Academy of Sciences of the USA, 100(12), 7051-7056 [Online]. Available: http://dx.doi.org/10.1073/pnas.1132112100.

Leloup, J. C., Gonze, D., \& Goldbeter, A. (1999). Limit cycle models for circadian rhythms based on transcriptional regulation in Drosophila and Neurospora. Journal of Biological Rhythms, 14(6), 433-448
Locke, J. C. W., Millar, A. J., \& Turner, M. S. (2005). Modelling genetic networks with noisy and varied experimental data: The circadian clock in Arabidopsis thaliana. Journal of Theoretical Biology, 234(3), 383-393 [Online]. Available: http://dx.doi.org/10.1016/j.jtbi.2004.11.038.

Ma, L., \& Iglesias, P. A. (2002). Quantifying robustness of biochemical network models. BMC Bioinformatics, 3, 38-50.

Merrow, M., Roenneberg, T., Macino, G., \& Franchi, L. (2001). A fungus among us: The Neurospora crassa circadian system. Seminars in Cell \& Developmental Biology, 12(4), 279-285 [Online]. Available: http://dx.doi.org/10.1006/scdb.2001.0255.

Morohashi, M., Winn, A. E., Borisuk, M. T., Bolouri, H., Doyle, J., \& Kitano, H. (2002). Robustness as a measure of plausibility in models of biochemical networks. Journal of Theoretical Biology, 216(1), 19-30 [Online]. Available: http://dx.doi.org/10.1006/jtbi.2002.2537.

Myers, M. P., Wager-Smith, K., Rothenfluh-Hilriker, A., \& Young, M. W. (1996). Light-induced degradation of TIMELESS and entrain-ment of the Drosophila circadian clock. Science, 271(5256), 1736-1740.

Panda, S., Hogenesch, J., \& Kay, S. (2002). Circadian rhythms from flies to human. Nature, 417, 329-335.

Park, J., Helfrich-Förster, C., Lee, G., Liu, L., Rosbash, M., \& Hall, J. (2000). Differential regulation of circadian pacemaker output by separate clock genes in Drosophila. Proceeding of the National Academy of Sciences of the USA, 97, 4087-4093.

Petri, B., \& Stengl, M. (1997). Pigment-dispersing hormone shifts the phase of the circadian pacemaker of the cockroach Leucophaea maderae. The Journal of Neuroscience, 17, 4087-4093.

Reppert, S. M., \& Weaver, D. R. (2000). Comparing clockworks: Mouse versus fly. Journal of Biological Rhythms, 15, 357-364.

Reppert, S. M., \& Weaver, D. R. (2001). Molecular analysis of mammalian circadian rhythms. Annual Review of Physiology, 63, 647-676.

Reppert, S. M., \& Weaver, D. R. (2002). Coordination of circadian timing in mammals. Nature, 418(6901), 935-941 [Online]. Available: http://dx.doi.org/10.1038/nature00965.

Roenneberg, T., Daan, S., \& Merrow, M. (2003). The art of entrainment. Journal of Biological Rhythms, 18, 183-194.

Ruoff, P., Vinsjevik, M., Monnerjahn, C., \& Rensing, L. (2001). The Goodwin model: Simulating the effect of light pulses on the circadian sporulation rhythm of Neurospora crassa. Journal of Theoretical Biology, 209(1), 29-42 [Online]. Available: http://dx.doi.org/10.1006/jtbi.2000.2239.

Salom, P. A., \& McClung, C. R. (2004). The Arabidopsis thaliana clock. Journal of Biological Rhythms, 19(5), 425-435 [Online]. Available: http://dx.doi.org/10.1177/0748730404268112.

Shearman, L. P., Sriram, S., Weaver, D. R., Maywood, E. S., Chaves, I., Zheng, B., et al. (2000). Interacting molecular loops in the mammalian circadian clock. Science, 288(5468), 1013-1019.

Smolen, P., Baxter, D. A., \& Byrne, J. H. (2001). Modeling circadian oscillations with interlocking positive and negative feedback loops. The Journal of Neuroscience, 21(17), 6644-6656.

Stelling, J., Gilles, E. D., \& Doyle, F. J., III. (2004). Robustness properties of circadian clock architectures. Proceeding of the National Academy of Sciences of the USA, 101(36), 13210-13215 [Online]. Available: http://dx.doi.org/10.1073/pnas.0401463101.

Stelling, J., Sauer, U., Szallasi, Z., Doyle, F. J., III, \& Doyle, J. (2004). Robustness of cellular functions. Cell, 118(6), 675-685 [Online]. Available: http://dx.doi.org/10.1016/j.cell.2004.09.008.

Stunners, S. M. (1998). Advanced modern control system theory and design. New York, NY: John Wiley \& Sons Inc.

Taylor, S., Gunawan, R., Gadkar, K., \& Doyle III, F. J., (2005). Biosens: A sensitivity analysis toolkit for Bio-SPICE. Available at http://www. chemengr.ucsb.edu/ ceweb/faculty/doyle/biosens/BioSens.htm.

To, T.-L., Henson, M., \& Doyle, F. J., III. (2005). Modeling the synchronization of biochemically coupled circadian oscillators. In Proceeding of International Conference Foundations of Systems Biology in Engineering.

Tyson, J. J., Hong, C. I., Thron, C. D., \& Novak, B. (1999). Simple model of circadian rhythms based on dimerization and proteolysis of PER and TIM. Biophysical Journal, 77, 2411-2417.

Ueda, H. R., Hagiwara, M., \& Kitano, H. (2001). Robust oscillations within the interlocked feedback model of Drosophila circadian rhythm. 
Journal of Theoretical Biology, 210(4), 401-406 [Online]. Available: http://dx.doi.org/10.1006/jtbi.2000.2226.

Varma, A., Morbidelli, M., \& Wu, H. (1999). Parametric sensitivity in chemical systems. New York, NY: Oxford University Press.

Williams, J. A., \& Sehgal, A. (2001). Molecular components of the circadian system in Drosophila. Brain Research Reviews, 63, 729-755.
Young, M., \& Kay, S. (2001). Time zones: A comparative genetics of circadian clocks. Nature Review Genetics, 2, 702-715.

Zeng, H., Qian, Z., Myers, M. P., \& Rosbash, M. (1996). A light-entrainment mechanism for the Drosophila circadian clock. Nature, 380(6570), 129-135. 